\title{
Extensive Air Showers with Unusual Spatial and Temporal Structure
}

\author{
Rashid Beisembaev ${ }^{1}$, Dmitriy Beznosko ${ }^{2, \star}$, Kanat Baigarin ${ }^{3}$, Ayan Batyrkhanov ${ }^{3}$, Elena Beisembaeva ${ }^{1}$, Oleg \\ Dalkarov ${ }^{1}$, Alexander lakovlev², Vladimi Ryabov ${ }^{1}$, Turlan Sadykov ${ }^{4}$, Sergei Shaulov${ }^{1}$, Marina Vildanova ${ }^{1}$, Tileubek \\ Uakhitov $^{3}$, and Valeriy Zhukov ${ }^{1}$ \\ ${ }^{1}$ P. N. Lebedev Physical Institute of the Russian Academy of Sciences, Moscow, Russia \\ ${ }^{2}$ Bard Early College New Orleans, New Orleans, USA \\ ${ }^{3}$ Nazarbayev University, Astana, KZ \\ ${ }^{4}$ LLP "Institute of Physics and Technology", Almaty, Kazakhstan
}

\begin{abstract}
.
The Extensive Air Shower (EAS) data collected by the Horizon-10T detector system has numerous events exhibiting the unusual spatial and temporal structure. These are events typically with two to four pulses of tens of ns wide that are present at distances above $300 \mathrm{~m}$ from the EAS axis, with these pulses being delayed by hundreds of ns. Each of such pulse groups were registered simultaneously by several detectors separated by up to a $\mathrm{km}$ from each other. The detectors brief overview and the analysis of simulated EAS by the CORSIKA software package are given in this article. The analysis indicates that such events with unusual structure cannot be formed by showers with EM and hadronic components only. This indicates a possibility for a new process observation beyond the standard model description in the energy range above $\sim 10^{18} \mathrm{eV}$.
\end{abstract}

\section{Introduction}

The Horizon-10T (H10T) [1] is an upgrade from the previous Horizon-T (HT) [2] detector system that was constructed at the Tien Shan High-altitude Science Station (TSHSS). The station is part of the P. N. Lebedev Physical Institute of the Russian Academy of Sciences and is located at a height of $\sim 3340 \mathrm{~m}$ above sea level near the city of Almaty, Kazakhstan. H10T is designed to study both the spatial and temporal distribution of the charged particles within the EAS with a time resolution of a few ns. It consists of ten charged particle detection points separated by distances up to $1.3 \mathrm{~km}$. The aerial view of the detector system is shown in Figure 1.

\section{H10T Detectors}

The H10T detector system consists of: plastic scintillator detectors [3] with Hamamatsu [4] R7723 or H6527 PMT, and glass detectors with R7723 PMT.

All Scintillator Detectors (SD) use polystyrene-based square-shaped cast scintillator with $1 \mathrm{~m}^{2}$ area and $5 \mathrm{~cm}$ thickness. The SDs in detection points $1-8$ use R7723 PMT assembly as photo-detector, and the far points 9 and 10 use H6527 that have higher gain and photosensitive area to compensate for signal loss in cables. The cable calibration $[1,5]$ shows how the signal changes with cable length. Detection points 1, 4, 5, 6 and 7 also have glassbased detectors (GD) together with the SD. Each GD uses

\footnotetext{
^e-mail: dmitriy.beznosko@dozory.us
}

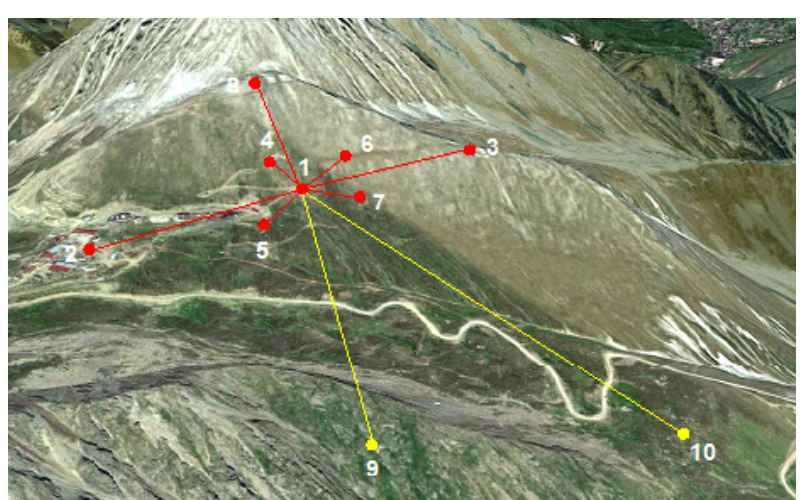

Figure 1. Aerial view of the Horizon-10T detector system.

$50 \mathrm{~cm} \times 50 \mathrm{~cm} \times 3 \mathrm{~cm}$ optical glass that is painted white using $\mathrm{TiO}_{2}$ and has a R7723 PMT assembly.

Each detector is calibrated for minimum ionizing particles signal using the background cosmic ray flux. The details of calibration can be found in [6].

\section{Data Acquisition System and Trigger Logic}

The H10T DAQ system consists of 3 CAEN [7] DT5730 analog to digital converter (ADC) units and custom control software.

One of the ADCs is designated as a main board and is used to form a hardware master trigger using double 


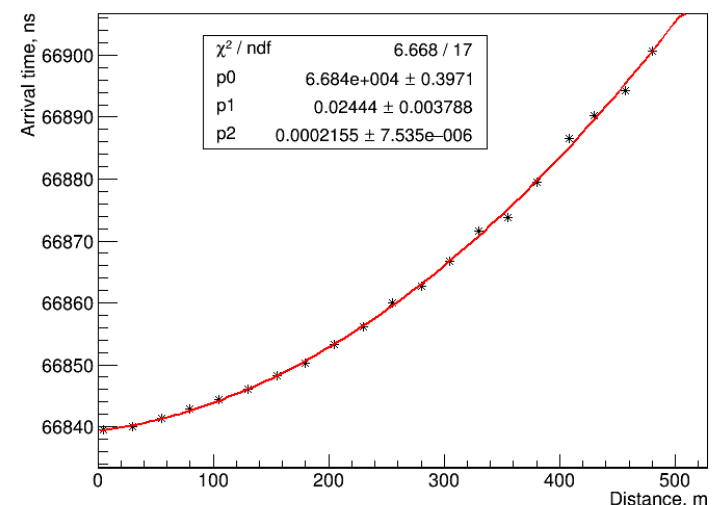

Figure 2. Arrival times at different distances from EAS axis at the observation level.

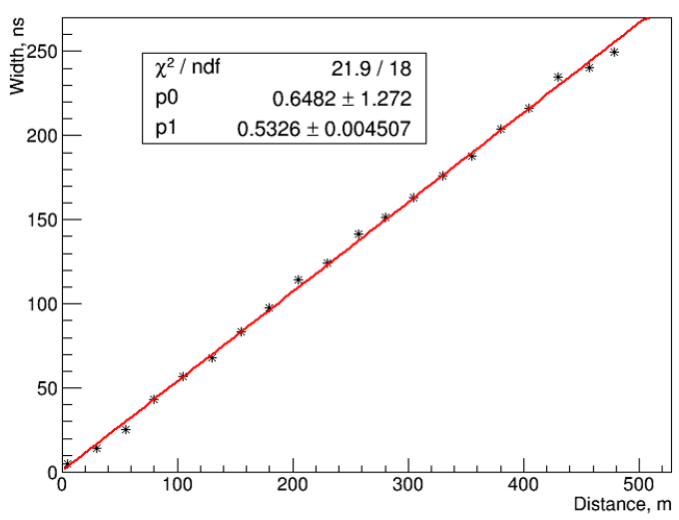

Figure 3. EAS disk width at different distances from axis at the observation level.

coincidence from detection points 5 and 6 (or 4 and 7). Then the trigger is fed to all ADCs. The same pulse is used for the synchronization that is recorded by the 8th channel of each ADC.

Offline triggers are applied to data before analysis. Minimally we require at least 4 detection points to have a signal at the level above 7 widths of the noise floor. Resultant rate of EAS registration intensity rate is $\sim 45$ events/day at the H10T detector system.

\section{Standard EAS Event Definition}

The choice is made to accept as a Standard Event (SE) [8] the showers as generated by the EAS simulation package CORSIKA [9]. The produced simulated events can be used as a reference and they also allow to assess various EAS properties. For illustration, Figure 2 shows the arrival time vs. distance from the axis of EAS disk at the observation level. Times are selected at 50\% of the particles passing through a simulated detector. Figure 3 displays the width of the EAS disk in ns, defined as the time between the $10 \%$ and $90 \%$ of particles at a detector. The

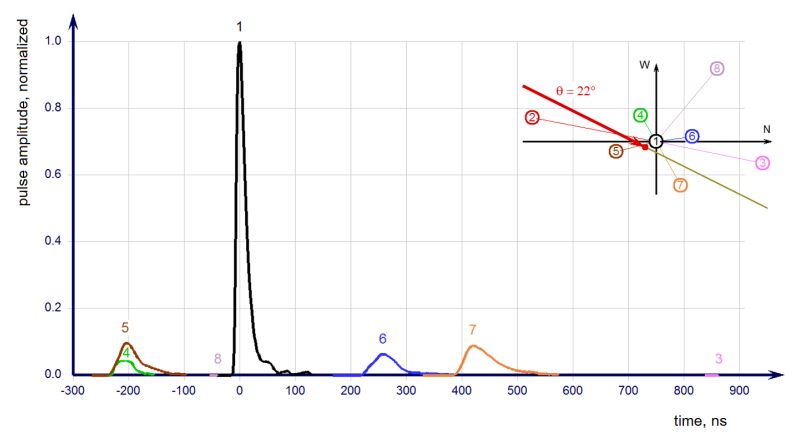

Figure 4. SE from H10T data.

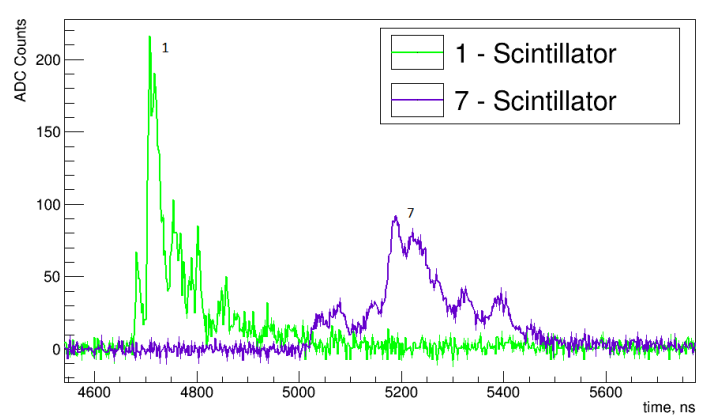

Figure 5. Unusual event from HT data.

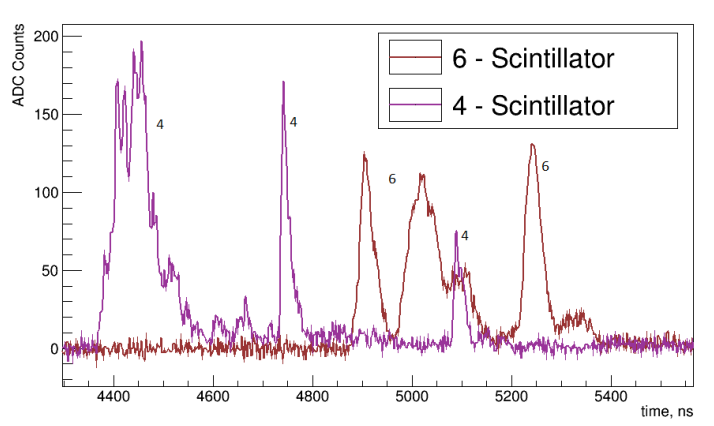

Figure 6. Unusual event from HT data.

figures have been fitted with quadratic and linear functions respectively.

The arrival time is used to obtain the direction of the primary for each EAS. But one can use the pulse width information in addition to the particle density distribution for the event reconstruction. This requires the detector time resolution $<10 \mathrm{~ns}$ for the width information to be used in the reconstruction. Figure 4 shows an event from data which corresponds to the definition of an SE and can be analyzed using pulse width information in addition to particle density and arrival time. Amplitudes of all pulses are normalized to the maximal one. 


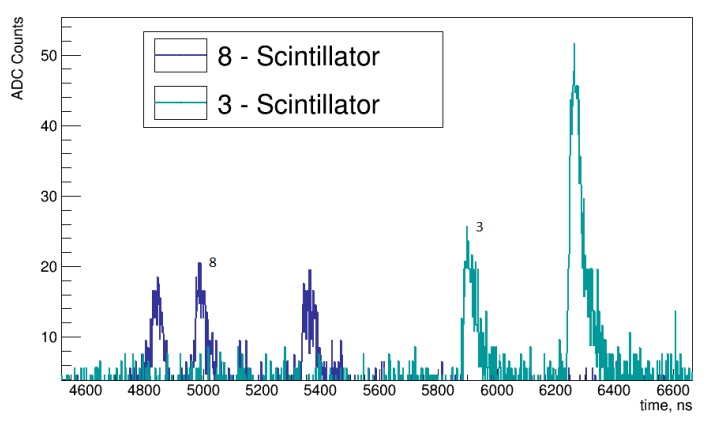

Figure 7. Unusual event from HT data.

\section{Unusual Event Definition}

The event shown in parts in Figures 5, 6 and 7 was detected on Jan. 262016 at 3:51 UTC and is fully shown and described in [8]. The axis of this event was between detection points 2 and 5 , closer to 2 . You can see how the separation between pulses increases with distance from the axis. We will use this example to define a class of Unusual Events (UE) as events that have multiple peaks (or modes) in several detectors. The main property of such events is that the time between peaks varies with the distance from the EAS axis.

The distance of detection points $1,7,6,4,3$ and 8 from the event axis grows in the order listed, and the time between modes increases following the same trend. This appears to be the property of all UEs detected.

\section{Constructing UE from Simulated SE}

Two possibilities of the UE formation from two standard EAS have been considered: firstly the EAS disk being delayed with respect to another as in Figure 8, and secondly the EAS disks arriving to the observation level simultaneously with their cores separated by some distance as shown in Figure 9.

To emulate how the possible pulses from each combination will look like, the simulated EAS disk from a $10^{17} \mathrm{eV}$ vertical proton is used. A simulated detector of 1 square meter area is placed at different distances from the EAS axis. Resulting histograms of particle numbers passing through the detector in time are produced.

First, we look at one EAS being delayed 100ns behind the first one. If the detector is placed $30 \mathrm{~m}$ from the common axis of these disks, the histogram of particle number in time is shown in Figure 10. Two clearly separated peaks are observed with high particle density as expected from the general disk shape and density distribution in it.

Figure 11 and Figure 12 show the particles distribution at $170 \mathrm{~m}$ and $290 \mathrm{~m}$ from the axis respectfully. We can see that the peaks are actually getting closer to each other at $170 \mathrm{~m}$ and the only reason separation is clearer at $290 \mathrm{~m}$ is due to the low particle density. Also peaks of higher widths are produced at larger distances. Thus, the observed UE can not be reproduced by two or more EAS disks delayed from each other.

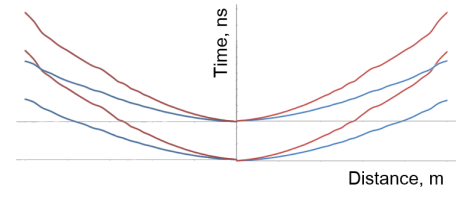

Figure 8. UE possible composition from one standard EAS delayed from another.

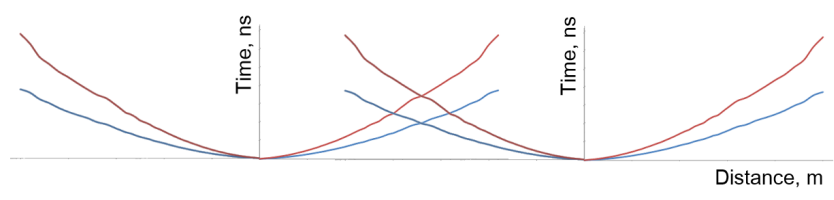

Figure 9. UE possible composition from two standard EAS next to each other.

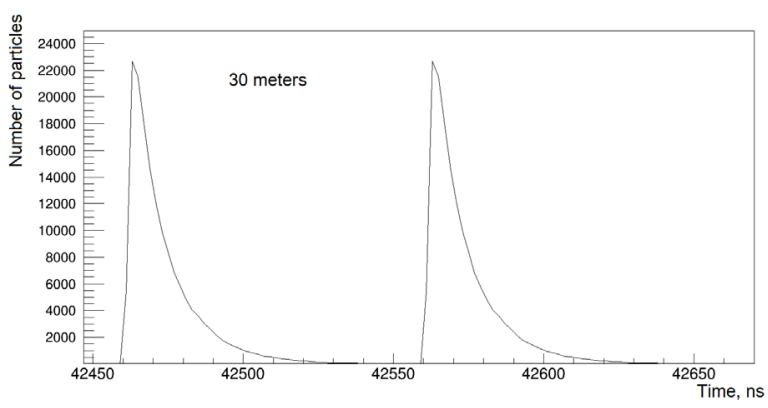

Figure 10. Two EAS disks with 100ns delay, detector is $30 \mathrm{~m}$ from axis.

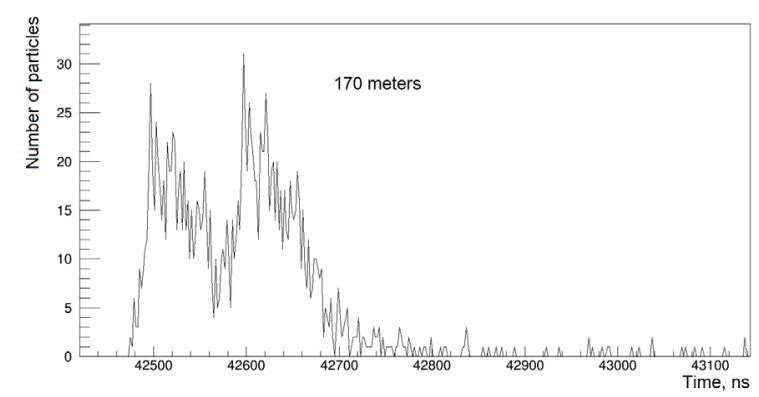

Figure 11. Two EAS disks with 100ns delay, detector is $170 \mathrm{~m}$ from axis.

Next we position two EAS disks side-by-side without any time delay between the parent particles. Figure 13 shows the particle time distribution in the detector placed $30 \mathrm{~m}$ from the axis of one EAS and $50 \mathrm{~m}$ from the axis of the other with the total separation between the axes being $80 \mathrm{~m}$. Then Figure 14 shows the particle time distribution in the detector placed $30 \mathrm{~m}$ from the axis of one EAS and $110 \mathrm{~m}$ from the axis of the other with the total separation between the axes being $140 \mathrm{~m}$. As we can see, the contribution from the second EAS is much lower then from first one due to the fast drop in particle density with distance 


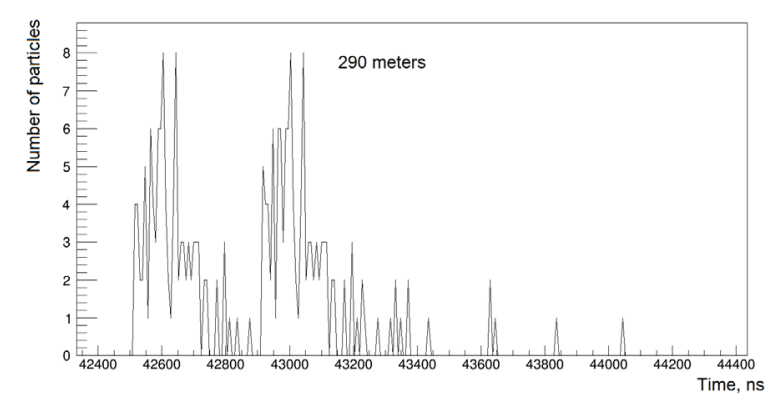

Figure 12. Two EAS disks with 100ns delay, detector is $290 \mathrm{~m}$ from axis.

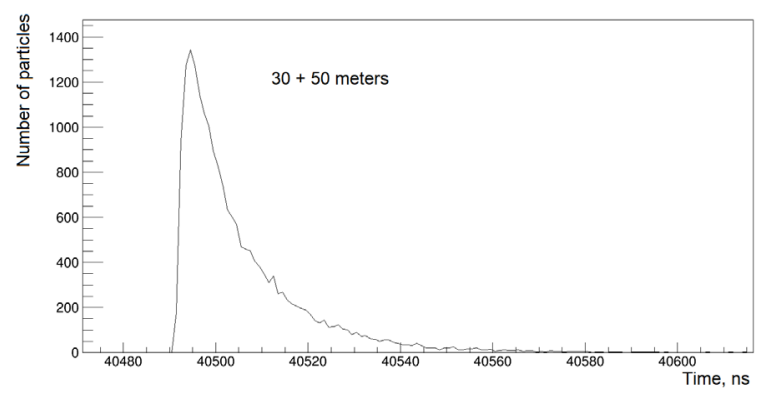

Figure 13. Two EAS disks side-by-side. Detector is $30 \mathrm{~m}$ from axis of one disk and $50 \mathrm{~m}$ from axis of another.

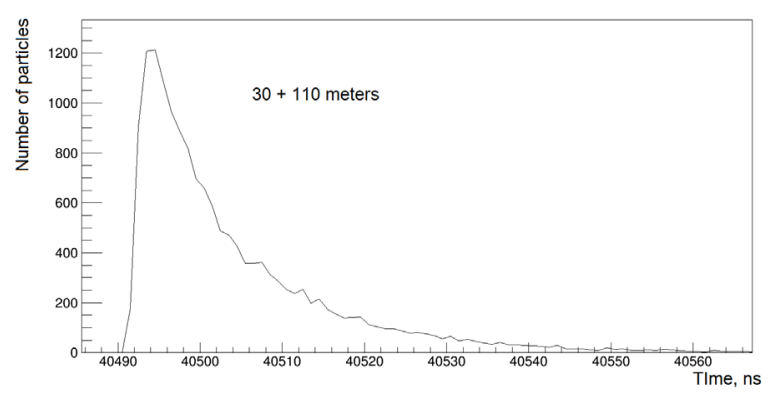

Figure 14. Two EAS disks side-by-side. Detector is $30 \mathrm{~m}$ from axis of one disk and $110 \mathrm{~m}$ from axis of another.

from the axis, so only a single widened pulse is detected. Thus this can not be how UEs are formed.

\section{Conclusion}

The Horizon-10T detector system is specifically designed for extended measurements for unusual events first reported by the Horizon-T detector. H10T is located at an altitude of about 3340 meters above sea level centered at geographical coordinates of $43^{\circ} 02^{\prime} 49^{\prime \prime} \mathrm{N}$ and $76^{\circ} 56^{\prime} 43^{\prime \prime}$ E. It has ten charged particle detection points, separated by hundreds of meters. Each point has scintillator detectors, some are also equipped with glass ones. Detectors are read out by Hamamatsu PMTs. A full waveform is digitized every 2 ns for a total of 5110 points.

Simulated EAS disks were used in an attempt to reproduce UE with a combination of SE. Two simple options - one disk delayed along the same axis, or two disks co-moving at some separation between their axes - do not give the same signal in a simulated detector as the unusual event data from H10T. The data analysis continues and more physics runs are planned. Far detectors (from center point 1) upgrade to larger areas is planned.

\section{Acknowledgments}

This work was funded in part by MES RK state-targeted program BR95236464.

\section{References}

[1] D Beznosko et al., arXiv:1803.08309 (2018)

[2] R U Beisembaev et al., arXiv:1605.05179 (2016)

[3] Dmitriy Beznosko et al., EPJ Web of Conferences 145, 19016 (2017)

[4] Hamamatsu Photonics, 314-5 Shimokanzo, Toyookavillage, Iwatagun, Shizuoka-ken, 438-0193 Japan http://www.hamamatsu.com

[5] T Beremkulov et al., arXiv:1608.04312, (2016)

[6] Dmitriy Beznosko et al., EPJ Web of Conferences 145, 11004, (2017)

[7] CAEN S.p.A. Via della Vetraia, 11, 55049 Viareggio Lucca, Italy http://www.caen.it

[8] Dmitriy Beznosko et al., EPJ Web of Conferences 145, 14001 (2017)

[9] D. Heck et al., Forschungszentrum Karlsruhe Report FZKA 6019 (1998) 